\title{
Rhamnogalacturonan lyase: A pectin modification enzyme of higher plants
}

\author{
Verónica-Alhelí Ochoa-Jiménez', Guillermo Berumen-Varela', Rigel Fernández-Valle, Martín-Ernesto, \\ Tiznado-Hernández*
}

Coordinación de Tecnología de Alimentos de Origen Vegetal, Centro de Investigación en Alimentación y Desarrollo, A. C., Carretera Gustavo Enrique Astiazarán Rosas No. 46, Colonia La Victoria

\#These authors contributed equally to this work

\section{A B S T R A C T}

\begin{abstract}
Plant cell wall is constituted by three main polysaccharides: cellulose, hemicellulose and pectin. The principal pectin domains comprise homogalacturonan, rhamnogalacturonan I (RG-I) and ramnogalacturonan II. Rhamnogalacturonan lyase (RGL) enzyme is capable of catalyzing the degradation of the RG-I backbone by a $\beta$-elimination mechanism. RGL enzyme have been studied in fungi and bacteria species, however, little data is available related with its function in plant biology. This review tries to fill the gaps on the knowledge about RGL enzyme. Here, we discuss our recent published work regarding the role of RGL in plants. In addition, we highlight results from different sequence analysis, RGL activity and function associated with plant development and fruit ripening, as well as its role in cell wall structure. The knowledge of this enzyme is essential to comprehend and elucidate its role in plant and fruit physiology.
\end{abstract}

Keywords: Enzyme; Domain; Phylogenetic tree; Plants; Rhamnogalacturonan lyase

\section{INTRODUCTION}

The plant cell wall is a complex matrix formed by polysaccharides, proteins, aromatic, and aliphatic compounds that surround and protects the cell from different environmental conditions (Caffall and Mohnen, 2009). Plant cell walls are classified based on the structural and functional differences in primary and secondary cell wall. The primary cell wall contains less cellulose and grater pectin in comparison with secondary walls. Primary wall contributes to physical integrity, cell adhesion and signal transduction (Caffall and Mohnen, 2009). Carbohydrates basically compose the primary cell wall, which are classified in three types: cellulose, hemicellulose and pectin. Among these components, pectins are the most plentiful polysaccharide of the primary cell wall. In this regard, monocots and dicots plants have pectins as constituents of their primary cell walls (Carpita and Gibeaut, 1993). Pectin are composed by several polymers, including: homogalacturonan (HG), rhamnogalacturonan-I (RG-I), rhamnogalacturonan-II (RG-II), apiogalacturonan and xylogalacturonan, among others (Harholt et al., 2010; Patova et al., 2014). HG is the most abundant polysaccharide in the pectin domain constituting more than 60\% (Caffall and Mohnen, 2009). HG is basically an unbranched homopolymer chain of $\alpha$-1,4-linked D-galacturonic acid moieties which can be methyl esterified at the carboxylic group. The most complex polysaccharide is RG-II, which makes up between $0.1 \%$ and $10 \%$ of pectin, depending of plant species. Further, it is made of sugars and linkage structures such as: apiose, aceric acid (3-C'- carboxy-5-deoxy-L-xylose), 2-O-methyl fucose, 2-O-methyl xylose, 3-deoxy-D-manno-2-octulosonic acid (kdo) and 3-deoxy-D-lyxo-2-heptulosaric acid. Besides, RG-II includes a diester bonds per boron atom between apiose units and in this way, two molecules can cross-link. The structure of this polymer is quite conserved among angiosperms and despite its low abundance, it is supposed to play an important role in the plant and cell physiology (Carpita, 1996).

On the other hand, RG-I is the second most abundant polysaccharide located in the cell wall (Yapo, 2011) and

\footnotetext{
${ }^{*}$ Corresponding author:

Tiznado-Hernández, Coordinación de Tecnología de Alimentos de Origen Vegetal, Centro de Investigación en Alimentación y Desarrollo, A. C., Carretera Gustavo Enrique Astiazarán Rosas No. 46. Colonia La Victoria. Código Postal 83304, México. Tel. +52 (662) 2892421 ;

Fax. +52 (662) 289 2400. E-mail: tiznado@ciad.mx 
represents 20 to $35 \%$ of the total pectin (Mohnen, 2008). RG-I exhibits high degree of diversity among cell type and number of sugars and it is composed of repeated units of a disaccharide constituted of L-rhamnose and D-galacturonic acid, linked by $\alpha-1,4$ glycosidic bonds.

The information associated with the pectin structure, can help to recognize the role of their components on plant physiology. However, in this review, we will focus on the RG-I structure of the cell wall.

\section{RG-I and its role on cell wall}

RG-I is a heavily branched polysaccharide constituted of a central chain of L-rhamnopyranosyl (L-Rhap) and D-galactopyranosyl (D-Galp) disaccharide residues. At least $20 \%$ of the L-Rhap residues are substituted with chains of L-arabinofuranosyl (L-Araf) and D-Galp (Dhillon et al., 2016). RG-I hold carbohydrate side chains of arabinan, galactan and arabinogalactan enclosed to the C4 carbon of the rhamnose moiety.

The structure of the RG-I arabinan and galactan chain differs depending upon the isolation source which can be apple (Schols et al., 1994), sugar beet (Sakamoto and Sakai, 1995), soybean (Nakamura et al., 2002; Huisman et al., 2001), persimmon (Duan et al., 2003) and potato (Bush et al., 2001; Skjøt et al., 2002). Moreover, previous studies have shown that RG-I side chains are developmentally and differentially regulated (Bush et al., 2001; Willats et al., 2001).

RG-I and RG-II are polysaccharides that are usually regarded as "hairy regions" of the plant cell wall. The natural complexity and composition of RG-I explains the need for a great number of enzymes needed to synthesize and degrade pectin (de Vries and Visser, 2001; Ridley et al., 2001). Vincken et al. (2003) suggested another model in which the HG side chains are attached to RG-I backbone, therefore, the enzymatic cleavage of RG-I backbone could have an important effect on the linkage of the pectin network and sensible effects on the pectin stability and structure.

Following this trend, Oomen et al. (2002) clearly suggested that RG-I may play an important function in cell wall firmness. The previous statement is based on immunocytochemical studies with galactan's and arabinan's antibodies which showed a drastic reduction in galactan and arabinan side-chains of RG-I in the wall of parenchyma cells in transformed potato plants carrying an RGL gene from Aspergillus. However, the epitopes of antibodies were located at cell corners in the middle lamella of transformed tubers contrary to their original location in the primary cell wall of parenchymal cells of the isogenic line (Oomen et al., 2002).
According to Harholtetal. (2010), only two glycosyltransferases (GTs) identified as ARABINANDEFICIENT1 (ARAD1) and XYLOGALACTURONANDEFICIENT1 (XGD1) were showed to be related with RG-I biosynthesis. Further, these enzymes are membrane proteins located in the Golgi apparatus. In a recent report by Buffetto et al. (2015), it was suggested that most likely several enzymes are needed for the RG-I side chain biosynthesis, which may be regulated during cell wall formation. However, the exact physiological function in the plant cell wall of RG-I is still unclear, including the specific role of each RG-I pectin structural domains (Buffetto et al., 2015).

RG-I has been related with cell wall mechanical properties, because the structure of galactan and arabinan contribute to cell wall firmness and elasticity, respectively (Lee et al., 2013). The complex structure of the RG-I and their conservation in plants, appears to affect the biological function of the plant cell wall. Nonetheless, more research is needed to understand its role on cell wall.

\section{Rhamnogalacturonan lyase enzyme}

According with Naran et al. (2007), RG-I is degraded by two specific endo-acting enzymes such as rhamnogalacturonan hydrolase (RGH) and rhamnogalacturonan lyase (RGL), also known as rhamnogalacturonan $\alpha$-Dgalactopyranosyluronide-(1,2)- $\alpha$-L-rhamnopyranosyl hydrolase and rhamnogalacturonan $\alpha$-L-rhamnopyranosyl$(1,4)-\alpha-\mathrm{D}$-galactopyranosyluronide lyase, respectively. RGL enzyme is responsible for cleaving the $\alpha-(1,4)$ glycosidic bonds between rhamnose and galacturonic acid of the RG-I main backbone through a $\beta$-elimination reaction, giving rise to a reducing end on one fragment, and an unsaturated ring on the non-reducing end of the polysaccharide (Mckie et al., 2001; McDonough et al., 2004) as shown in (Fig. 1).

The activity of pectin lyase and particularly the RGL is quantified by the increase in optical density at $235 \mathrm{~nm}$ because the $\beta$-elimination mechanism generate the 4,5 -unsaturated galacturonic acid. The value of the molar extinction coefficient of the reaction product at $235 \mathrm{~nm}$ is $5.5 \times 10^{3} \mathrm{M}^{-1} \mathrm{~cm}^{-1}$. However, although the unsaturated uronic acid is usually detected by absorption at $235 \mathrm{~nm}$, when it is present in nanomole quantities, it is challenging to quantify spectrophotometrically (Naran et al., 2007; Charnock et al., 2002). Furthermore, the creation of double bound between $\mathrm{C} 4$ and $\mathrm{C} 5$ at the non-reducing end of the uronic acid is sufficient to recognize the work of a lyase, but not enough to confirm whether it acts over either RG-I or over polygalacturonic acid.

Silva et al. (2011) developed the enzyme assay conditions for the quantification of an RGL from Bacillus licheniformis 


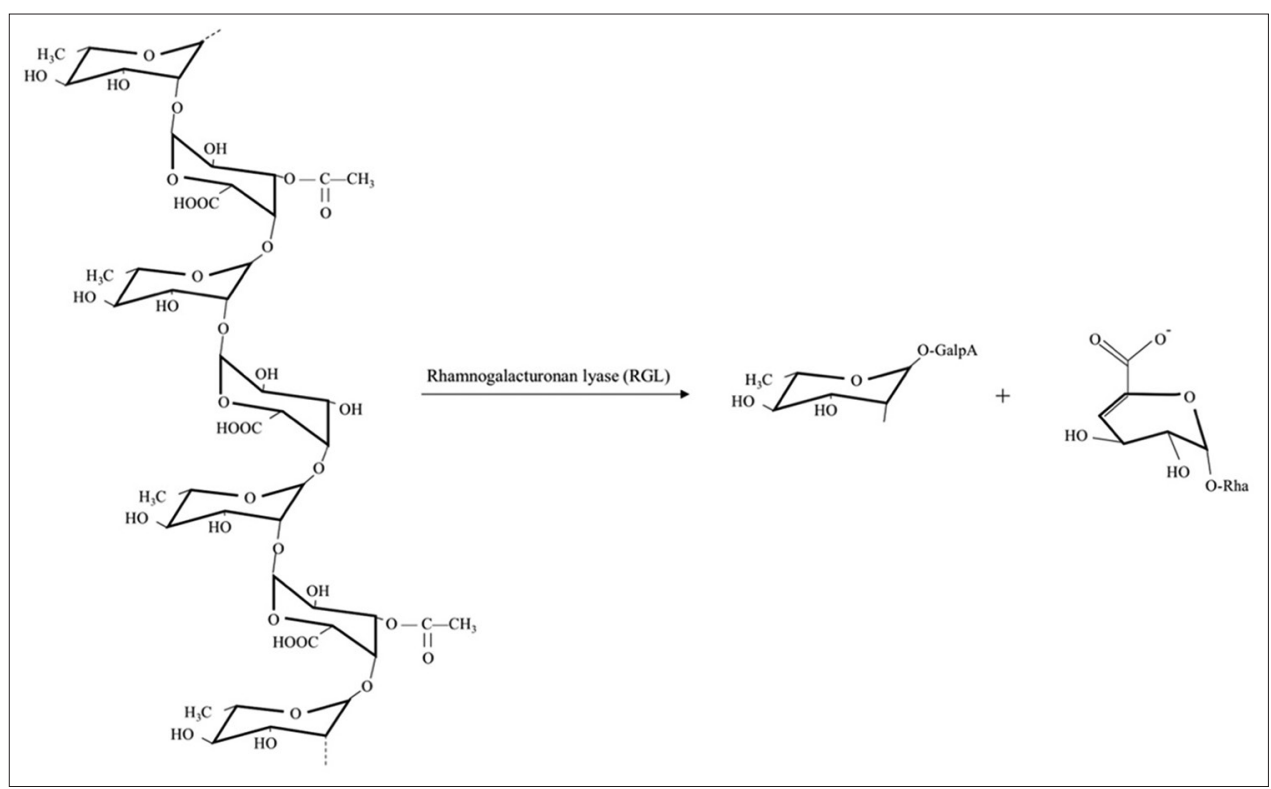

Fig 1. $\beta$-elimination mechanism of RGL on the RG-I backbone structure. The RGL enzymes cleaves the $\alpha-(1,4)$ of the glycosidic bonds between rhamnose and galacturonic acid producing GalpA oligomers at the non-reducing end and Rhap residue at the reducing end.

(DSM13) using potato rhamnogalacturonan as substrate. The best parameters were $61^{\circ} \mathrm{C}, \mathrm{pH} 8.1$ and $2 \mathrm{mM}$ of $\mathrm{Ca}^{+2}$ and $\mathrm{Mn}^{+2}$, being both ions essential for enzyme activity. Silva et al. (2016) summarized the optimal conditions for the eight kinds of endo and exo lyases in microorganisms like bacteria and fungi. The YesX exo-RG-I lyase originates unsaturated disaccharides as final product, additionally, it seems to be more active on pure sources of RG-I, like RG-I from potato, as compared with RG-I mixed with other polysaccharides (Silva et al., 2016). Reaction conditions of PL4 and PL11 enzymes also differ with respect to the optimal reaction conditions as stated by Silva et al. (2016). These authors revealed that the $\mathrm{pH}$ ideal of the PL11 enzymes is 8 or higher, while that of PL4 enzymes is 6 .

Few reports analyzing the RGL activity in plants have been pubished. In this sense, Stratilova et al. (1998) evaluated the RGL activity in carrot roots and leaves using $0.1 \%$ rhamnogalacturonan in $\mathrm{pH} 4.0$ acetate buffer as a substrate, detecting only low activity in roots.

In cotton (Gossypium birsutum, L.) cotyledons, Naran et al. (2007) labeled RGL oligomers with a fluorescent tag and injected them into the intercellular spaces, during the cell expansion of cotyledons. The activity registered for the enzyme at the intercellular spaces was very low and could be detected most easily when not more than $0.03 \mathrm{nmol}$ of substrate was injected and incubated in vivo. RGL activity was maximum in 3 to 4 day old cotyledons and progressively declined during the slow-down of expansion through the next 2-3 days. They also found that this activity was detected when linear rhamnogalacturonan oligomers (GR) consisting of 12-20 residues long with GalA and Rha at the non-reducing and reducing end respectively (cleaving Rha-GalA glycosidic linkages) were inserted into intercellular spaces by infiltration technique, showing that such activity was not induced by wounding (Naran et al., 2007).

On the other hand, in a recent paper by our investigation group, the RGL activity on leaves, roots, flowers and during fruit development and ripening were evaluated spectrophotometrically in transgenic tomato lines harboring the Solyc11g011300 gene, which encodes a RGL isoenzyme, under the control of the CaMV35S promoter as well as in the isogenic lines tomatoes using $50 \mathrm{mM}$ RG-I from potato (Megazyme T-RHAM 09/02) in pH 8.5 Tris- $\mathrm{HCl}$ buffer as a substrate and $2 \mathrm{mM}$ of $\mathrm{CaCl}_{2}$. It was found RGL activity in all lines and tissues tested. Transgenic tomato lines showed lower values of activity in leaves, roots and at mature green, turning and red ripe tomatoes than isogenic line. Besides, the highest RGL activity was recorded at 20 days after anthesis in plants harboring the RGL construct (Ochoa-Jiménez et al., 2018).

Functional domain and phylogenetic analysis of RGL The prediction of protein domains is required for understanding the putative function of a protein at biochemical and molecular level. In order to recognize the mechanism of the RGL enzyme in plants, we carried out a functional domain analysis with the amino acid RGL sequences of different plant species and Aspergillus aculeatus (which is the most extensive studied fungi with sequences of RGL in the genome) using Pfam software. 
The comparison between RGL sequences from plant species with a RGL from fungi, showed a low degree of similarity, exhibiting only a $23-26 \%$ of identity between plant species and A. aculeatus. (Table 1).

In agreement with this, Jensen et al. (2010) reached the conclusion that PL4 enzymes from plants homologues showed a $25 \%$ of identity, while bacteria homologues showed up to $44 \%$ of sequence identity to a RGL4 sequence from $A$. aculeatus. Besides, three functional domains were found in all plant species evaluated, a rhamnogalacturonan lyase domain (Rhamnogal_lyase) and two C-terminal domains named Polysaccharide lyase family 4, which is domain II (fn3_3) and Polysaccharide lyase family 4 , representing domain III (CBM-like) as it can be observed in (Fig. 2). The domain I (Rhamnogal_lyase domain) contains the catalytic active site and substrate binding residues (Jensen et al., 2010). This domain belongs to the $\beta$-Sandwich class, and it is located in the N-terminal and predicted to contain the catalytic site, consisting of two conserved residues of Lys150 and His210. The FnIII-like domain (domain II) displays a Fibronectin-III type fold and has a small beta-sandwich subdomain similar to the prohormone/propeptide processing enzyme carboxypeptidase gp180, and generally acts as a linker domain. The domain III, the CBM-like (carbohydrate binding module) domain contains a jelly roll beta-sandwich fold and hosts two sulfate ions and a hexa-coordinated calcium ion loop structure (Silva et al., 2016). This C-terminal domain is localized distal to the active site, and hence perhaps does not play a direct

Table 1: Comparison between amino acid sequences of RGL from plants species versus Aspergillus aculeatus

\begin{tabular}{llcc}
\hline Accesion number & Species & $\begin{array}{c}\text { Query } \\
\text { cover (\%) }\end{array}$ & Identity (\%) \\
\hline XP_009803162.1 & Nicotiana sylvestris & 38 & 26 \\
XP_003526528.1 & Glycine max & 37 & 26 \\
XP_016556229.1 & Capsicum annum & 38 & 24 \\
XP_015073522.1 & Solanum penelli & 38 & 23 \\
XP_002271814.3 & Vitis Vinifera & 38 & 23 \\
NP_195516.2 & Arabidopsis thaliana & 38 & 25 \\
\hline
\end{tabular}

function in the catalysis (McDonough et al., 2004; Ridley et al., 2001). The possible proton abstractor is suggested to be the Lys150 residue, acting at C5 position of the GalpA residue whereas the His 210 acts as a proton donor for the leaving group. The probable reason because the catalytic activity does need of a calcium ion for stabilization is because this reaction does not necessitate the formation of a carbanion intermediate (Rexová-Benková and Markoviĉ, 1976). On the other hand, PL11 family contains the two C-terminal domains found in plant species with a large degree of identity among them (Silva et al., 2016). In the case of family PL11, there are two enzymes in this family, endolytic and exolytic, known as YesW and YesX, respectively. These enzymes possess two domains that are the $\mathrm{N}$-terminal domain and a $\beta$-propeller domain, binding the substrate to the opposite end of the last domain mentioned. Moreover, one $\mathrm{Mn}^{2+}$ ion is positioned at one end of the propeller that is probably the substrate-binding site (Garron and Cygler, 2010).

The degree of similarity between the RGL organisms from plant, bacteria and fungi is reported in a maximum likelihood tree based on amino acid sequences deposited in NCBI and Interpro databases using MEGA 7.0 software (Fig. 3). In this sense, we found that the distance between the fungi and plants are bigger than bacteria compared to fungi, forming three different groups, which clearly demonstrate the microorganisms are evolutionary closer. Furthermore, there is a low identity between plants and microorganisms as shown in Table 1. Perhaps the diversity in RGL enzymes of plants will be found to be large taking into account the wide diversity of compositions on primary cell walls from organs leaves, fruits, steams and roots.

\section{Role of RGL genes in plant physiology}

The role of RGL in different plant physiology phenomena including root differentiation and pollen tube growth requires more investigation (Sénéchal et al., 2014). Some studies mentioned that RGL plays a function in cell expansion phenomena, growth and plant development

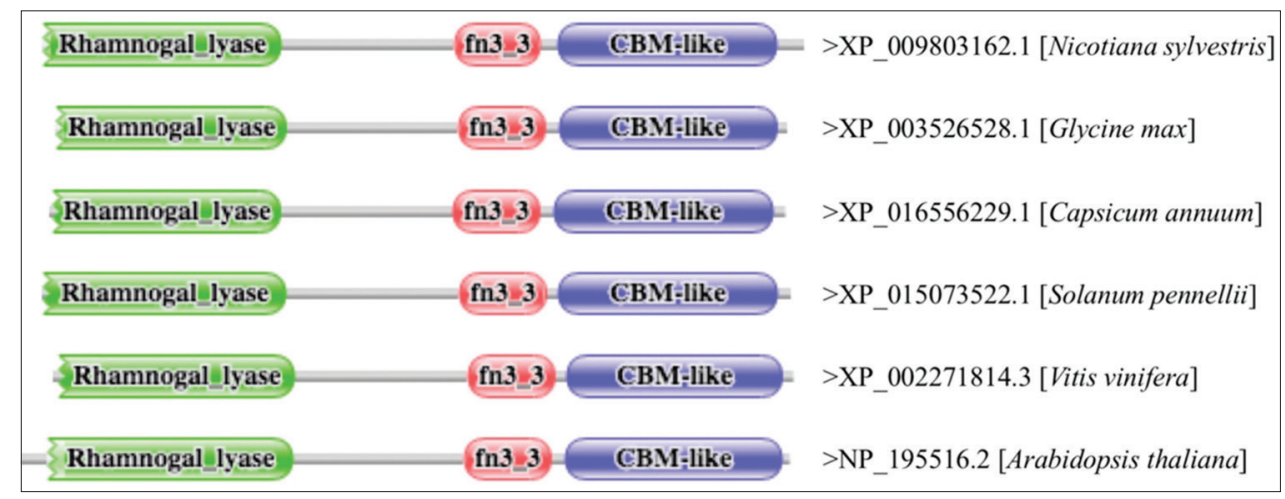

Fig 2. Functional domain analysis of Rhamnogalacturonan lyase proteins in plants. Green color represents RGL domain, red color the Fn III domain and purple CMB-like domain (Carbohydrate module binding). 


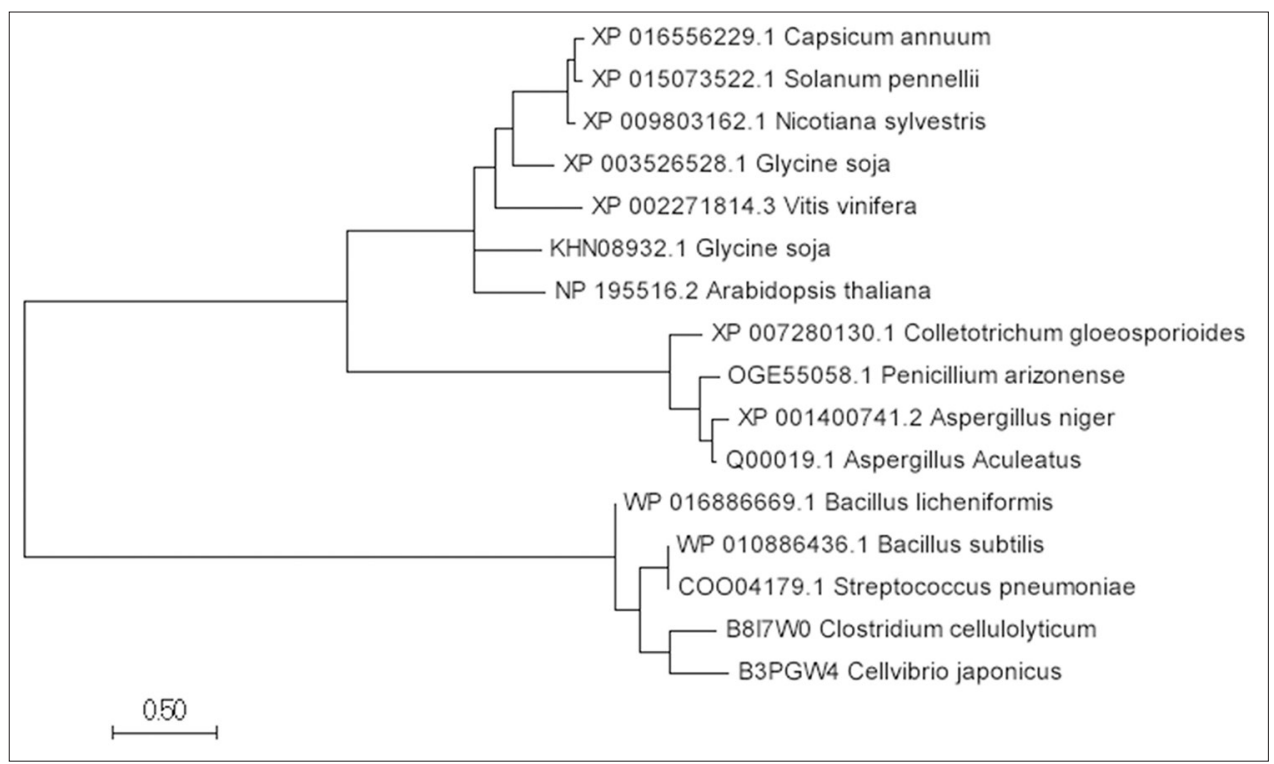

Fig 3. A maximum likelihood phylogenetic tree of RGL from different organisms (bacteria, fungi and plants) based on amino acid sequences. RGL amino acid sequences were aligned and analyzed using MEGA 7.0 software. The identification number (ID) and name of the organisms are shown in the figure.

(Naran et al., 2007) and softening during fruit ripening (Vicente et al., 2007). Oomen et al. (2002) created transgenic potato plants overexpressing a RGL gene encoding $A$. aculeatus, using the granule bound starch synthase promoter to drive the expression in potato tubers. Transgenic plants produced morphologically distorted tubers and pollen with low fertility. Besides, other abnormality presented was the gross changes in the cell wall composition including a reduction in RG-I and increase in HG. In a study carried out by Molina-Hidalgo et al. (2013) a putative RGL encoding gene designated as FaRGlyase1 was functionally characterized on strawberry fruits (Fragaria $\times$ ananassa). The results of that investigation showed a high expression levels of FaRGlyase1 gene in the fruit receptacle, alongside with ripening. Further, the FaRGlyase 1 gene was silenced via agroinfiltration with Agrobacterium cells harbouring a RNA interference construct. Light and electron microscopy of FaRGLyase1-silenced fruits, revealed that the middle lamella showed a higher degree of cell-wall integrity which indicates that the cell-wall showed lower alterations as compared with the control, showing that the RGL gene was involved in the pectin degradation existent in the middle lamella. These findings, strongly suggested that the FaRGlyase 1 might play a main role in the fruit softening process during strawberry post-harvest life.

Since last decade, RNA sequencing (RNA-seq) has been a suitable tool to provide understandings concerning gene expression patterns. In this regard, in a published transcriptome of mango mesocarp, it was reported one RGL gene differentially expressed (5-fold) in ripe mango comparing with mature green, which is $57 \%$ identical to the strawberry RGL enzyme previously mentioned
(Dautt-Castro et al., 2015). In this sense, a sequence analysis performed by the authors of this manuscript revealed that the tomato RGL gene, Solyc11g011300 is more than 65\% similarity to the strawberry RGL gene (FaRGlyase1).

Seven homologous genes to RGL from fungi have been found in the Arabidopsis thaliana genome sequences (http:/ / cellwall.genomics.purdue.edu). In addition, the tomato genome contains 13 genes encoding RGL isoenzymes (https:/ / solgenomics.net) which not all of them have been functionally tested. Berumen-Varela et al. (2017) analyzed all the promoter sequences from RGL genes of Solanum lycopersicum and Arabidopsis thaliana by bioinformatics. As a result of that investigation, cis-acting elements from RGL tomato promoters were classified in several groups, the elements identified in the RGL sequences from tomato indicate that RGL genes might play a role associated with ethylene, pollen and flower development, auxin and abscisic acid signaling, pathogen attack among others. In Arabidopsis the cis-acting elements have been related with flower development, abiotic stress, cellular metabolism, etc. These in silico analyses showed the importance of the RGL in plant physiology, however, further experimental studies were performed by our research group in order to support these conclusions. Trillo-Hernández (2016) evaluated the expression profiles of Solyc04g076660, Solyc07g046630 and Solyc11g011300 genes during tomato fruit development and ripening. The findings of that research concluded that Solyc04g076660 and Solyc04g076630 genes are involved in the early stages of tomato fruit development in which it is growing mainly by cell expansion and the Solyc11g011300 gene plays a role in the fruit softening phenomena during tomato fruit ripening. 
Furthermore, Jiménez-Maldonado et al. (2018) assessed the effect of RG-I fragments from potato on the defense mechanism in tomato fruits. Two fragments of RG-I with a molecular weight of 20 and $16 \mathrm{kDa}$ (F-1 and F-2 fragments respectively) induced the enzymatic activity of $\beta$-1,3-glucanase at $6 \mathrm{~h}$ after treatment. The F-1 fragment increased the chitinase and peroxidase activity after $48 \mathrm{~h}$ of exposure and F-2 fragment induce the chitinase and peroxidase activity at $0.5 \mathrm{~h}$ after applied the treatments. The findings of this research demonstrated that different fragment of RG-I can induce the defense mechanism of fruits at different time after treatment application.

Berumen-Varela et al. (2018) evaluated by qRT-PCR the expression levels of a tomato RGL gene, Solyc11g011300, during plant development and fruit ripening. The findings of this experiment showed a high expression levels in flowers and in red ripe tomatoes. Additionally, BerumenVarela et al. (2018) created transgenic tomato plants expressing the $\beta$-glucuronidase (GUS) reporter gene under the transcriptional control of two different lengths of the promoter of a tomato RGL gene, Solyc11g011300, to characterize its transcriptional regulation during plant development and fruit ripening. The results demonstrated that -504 and $-776 \mathrm{bp}$ of the RGL promoter are able to drive the GUS expression at pollen grains, pollen tube elongation, flowers and during tomato fruit ripening. Taken together, the results of this experiment lead to the conclusion that the promoter of the Solyc11g011300 gene is transcriptionally active and contains cis-acting elements conferring the organ-specificity in tomato.

Further, in order to investigate the role of the coding region of the Solyc11g011300 gene, Ochoa-Jiménez et al. (2018) created transgenic tomato plants overexpressing the RGL gene under the control of the CaMV35S promoter. The authors of that research evaluate the expression levels of different RGL genes, enzymatic activity of RGL and alterations in tissues and fruits of tomato plants during plant development and fruit ripening. The results of that investigation showed that the Solyc11g011300 gene is active in tomato plant and the overexpression of the RGL gene inhibits pollen germination and viability, and also affects the fruit yield due to a low number of seeds and fruits observed in transgenic lines compared with the isogenic line. In addition, transgenic lines showed an increase in the firmness of tomato fruits, delaying the fruit ripening and extending the postharvest shelf life.

Collectively, it can be concluded from the results of the investigations of Solyc11g011300 gene, that RGL is involved in flower and tomato fruit softening and ripening.

\section{CONCLUSIONS}

The recent work performed in plants provides new insights into the functions of the RGL enzyme in plants. Based on the most recent information showed in this review, the RGL enzymes could play a key role during pollen tube growth, defense mechanism against pathogen attack and it is involved in fruit softening and ripening phenomena.

\section{ACKNOWLEDGEMENTS}

This study was financially supported from the grant number 177248 of the National Council of Science and Technology (CONACYT) of Mexico.

\section{Authors Contributions}

Verónica Alhelí Ochoa-Jiménez: Write the entire manuscript, analyzed the phylogenetic tree of RGL from different organisms and recompilation and interpretation of data.

Guillermo Berumen-Varela: analyzed and modeled of $\beta$-elimination method, funcional domain analysis and compilation of aminoacid sequence.

Rigel Fernández-Valle: recompilation and interpretation of data.

Martín Ernesto Tiznado-Hernández: Contributed with the idea and knowledge of this investigation, review the entire manuscript and analyzed all the data.

\section{REFERENCES}

Berumen-Varela, G., V. A. Ochoa-Jiménez, A. Burgara-Estrella, E. A. Trillo-Hernández, Á.J. Ojeda-Contreras, A. OrozcoAvitia, M. Rivera-Domínguez, R. Troncoso-Rojas, R. BáezSañudo, T. Datsenka and M. E. Tiznado-Hernández. 2018. Functional analysis of a tomato (Solanum lycopersicum L.) rhamnogalacturonan lyase promoter. J. Plant Physiol. 229: 175-184.

Berumen-Varela, G., M. Rivera-Domínguez, R. TroncosoRojas, R. Báez-Sañudo and M. E. Tiznado-Hernández. 2017. Physiological function of rhamnogalacturonan lyase genes based in the analysis of cis-acting elements located in the promoter region. Res. J. Biotechnol. 12: 77-108.

Buffetto, F., V. Cornuault, M. G. Rydahl, D. Ropartz, C. Alvarado, V. Echasserieau, S. Le Gall, B. Bouchet, O. Tranquet and Y. Verhertbruggen. 2015. The deconstruction of pectic rhamnogalacturonan I unmasks the occurrence of a novel arabinogalactan oligosaccharide epitope. Plant Cell Physiol. 56: 2181-2196.

Bush, M. S., M. Marry, M. I. Huxham, M. C. Jarvis and M. C. McCann. 2001. Developmental regulation of pectic epitopes during potato tuberisation. Planta. 213: 869-880.

Caffall, K. H. and D. Mohnen. 2009. The structure, function, and 
biosynthesis of plant cell wall pectic polysaccharides. Carbohydr. Res. 344: 1879-1900.

Carpita, N. C. 1996. Structure and biogenesis of the cell walls of grasses. Ann. Rev. Plant Biol. 47: 445-476.

Carpita, N. C. and D. M. Gibeaut. 1993. Structural models of primary cell walls in flowering plants: Consistency of molecular structure with the physical properties of the walls during growth. Plant $\mathrm{J}$. 3: 1-30.

Charnock, S. J., I. E. Brown, J. P. Turkenburg, G. W. Black and G. J. Davies. 2002. Convergent evolution sheds light on the anti- $\beta$-elimination mechanism common to family 1 and 10 polysaccharide lyases. Proc. Natl. Acad. Sci. 99: 12067-12072.

Dautt-Castro, M., A. Ochoa-Leyva, C. A. Contreras-Vergara, M. A. Pacheco-Sanchez, S. Casas-Flores, A. Sanchez-Flores, D. N. Kuhn and M. A. Islas-Osuna. 2015. Mango (Mangifera indica L.) cv. Kent fruit mesocarp de novo transcriptome assembly identifies gene families important for ripening. Front. Plant Sci. 6: 62.

de Vries, R. P. and J. Visser. 2001. Aspergillus enzymes involved in degradation of plant cell wall polysaccharides. Microbiol. Mol. Biol. Rev. 65: 497-522.

Dhillon, A., V. O. Fernandes, F. M. Dias, J. A. Prates, L. M. Ferreira, C. M. Fontes, M. Centeno and A. Goyal. 2016. A new member of family 11 polysaccharide lyase, rhamnogalacturonan lyase (CtRGLf) from Clostridium thermocellum. Mol. Biotechnol. 58: 232-240.

Duan, J., X. Wang, Q. Dong, J. N. Fang and X. Li. 2003. Structural features of a pectic arabinogalactan with immunological activity from the leaves of Diospyros kaki. Carbohydr. Res. 338: 1291-1297.

Garron, M. L. and M. Cygler. 2010. Structural and mechanistic classification of uronic acid-containing polysaccharide lyases. Glycobiology. 20: 1547-1573.

Harholt, J., A. Suttangkakul and H. V. Scheller. 2010. Biosynthesis of pectin. Plant Physiol. 153: 384-395.

Huisman, M. M., L. P. Brüll, J. E. Thomas-Oates, J. Haverkamp, H. A. Schols and A. G. Voragen. 2001. The occurrence of internal $(1 \rightarrow 5)$-linked arabinofuranose and arabinopyranose residues in arabinogalactan side chains from soybean pectic substances. Carbohydr. Res. 330: 103-114.

Jensen, M. H., H. Otten, U'. Christensen, T. V. Borchert, L. L. Christensen, S. Larsen and L. L. Leggio. 2010. Structural and biochemical studies elucidate the mechanism of rhamnogalacturonan lyase from Aspergillus aculeatus. J. Mol. Biol. 404: 100-111.

Jiménez-Maldonado, M. I., M. E. Tiznado-Hernández, A. RascónChu, E. Carvajal-Millán, J. Lizardi-Mendoza and R. TroncosoRojas. 2018. Analysis of rhamnogalacturonan I fragments as elicitors of the defense mechanism in tomato fruit. Chil. J. Agric. Res. 78: 339-349.

Lee, K. J., V. Cornuault, I. W. Manfield, M. C. Ralet and J. P. Knox. 2013. Multi-scale spatial heterogeneity of pectic rhamnogalacturonan I (RG-I) structural features in tobacco seed endosperm cell walls. Plant J. 75: 1018-1027.

McDonough, M. A., R. Kadirvelraj, P. Harris, J. C. Poulsen and S. Larsen. 2004. Rhamnogalacturonan lyase reveals a unique three-domain modular structure for polysaccharide lyase family 4. FEBS Lett. 565: 188-194.

Mckie, V. A., J. P. Vincken, A. G. Voragen, L. A. Van den broek, E. Stimson and H. J. Giilbert. 2001. A new family of rhamnogalacturonan lyases contains an enzyme that binds to cellulose. Biochem. J. 355: 167-177.
Mohnen, D. 2008. Pectin structure and biosynthesis. Curr. Opin. Plant Biol. 11: 266-277.

Molina-Hidalgo, F. J., A. R. Franco, C. Villatoro, L. MedinaPuche, J. A. Mercado, M. A. Hidalgo, A. Monfort, J. L. Caballero, J. Muñoz-Blanco and R. Blanco-Portales. 2013. The strawberry (Fragariaxananassa) fruit-specific rhamnogalacturonate lyase 1 (FaRGLyase1) gene encodes an enzyme involved in the degradation of cell-wall middle lamellae. J. Exp. Bot. 64: 1471-1483.

Nakamura, A., H. Furuta, H. Maeda, T. Takao and Y. Naganatsu. 2002. Structural studies by stepwise enzymatic degradation of the main backbone of soybean soluble polysaccharides consisting of galacturonan and rhamnogalacturonan. Biosci. Biotechnol. Biochem. 66: 1301-1313.

Naran, R., M. L. Pierce and A. J. Mort. 2007. Detection and identification of rhamnogalacturonan lyase activity in intercellular spaces of expanding cotton cotyledons. Plant J. 50: 95-107.

Ochoa-Jiménez, V. A., G. Berumen-Varela, A. Burgara-Estrella, J. A. Orozco-Avitia, Á. J. Ojeda-Contreras, E. A. Trillo-Hernández, M. Rivera-Domínguez, R. Troncoso-Rojas, R. Báez-Sañudo, T. Datsenka and M. E. Tiznado-Hernández. 2018. Functional analysis of tomato rhamnogalacturonan lyase gene Solyc11g011300 during fruit development and ripening. J. Plant Physiol. 231: 31-40.

Oomen, R. J., C. H. Doeswijk-Voragen, M. S. Bush, J. P. Vincken, B. Borkhardt, L. A. Van Den Broek, J. Corsar, P. Ulvskov, A. G. Voragen and M. C. McCann. 2002. In muro fragmentation of the rhamnogalacturonan I backbone in potato (Solanum tuberosum L.) results in a reduction and altered location of the galactan and arabinan side-chains and abnormal periderm development. Plant J. 30: 403-413.

Patova, O., V. Golovchenko and Y. S. Ovodov. 2014. Pectic polysaccharides: Structure and properties. Russ. Chem. Bull. 63: 1901-1924.

Rexová-Benková, L'. and O. Markoviĉ. 1976. Pectic enzymes. Adv. Carbohydr. Chem. Biochem. 33: 323-385.

Ridley, B. L., M. A. O'Neill and D. Mohnen. 2001. Pectins: Structure, biosynthesis, and oligogalacturonide-related signaling. Phytochemistry. 57: 929-967.

Sakamoto, T. and T. Sakai. 1995. Analysis of structure of sugar-beet pectin by enzymatic methods. Phytochemistry. 39: 821-823.

Schols, H. A., M. Mutter, A. G. Voragen, W. M. Niessen, R. A. van der Hoeven, J. van der Greef and C. Bruggink. 1994. The use of combined high-performance anion-exchange chromatographythermospray mass spectrometry in the structural analysis of pectic oligosaccharides. Carbohydr. Res. 261: 335-342.

Sénéchal, F., C. Wattier, C. Rustérucci and J. Pelloux. 2014. Homogalacturonan-modifying enzymes: Structure, expression, and roles in plants. J. Exp. Bot. 65: 5125-5160.

Silva, I. R., C. Jers, A. S. Meyer and J. D. Mikkelsen. 2016. Rhamnogalacturonan I modifying enzymes: An update. New Biotechnol. 33: 41-54.

Silva, I. R., D. M. Larsen, A. S. Meyer and J. D. Mikkelsen. 2011. Identification, expression, and characterization of a novel bacterial RGI Lyase enzyme for the production of bio-functional fibers. Enzym. Microbial Technol. 49: 160-166.

Skjøt, M., M. Pauly, M. S. Bush, B. Borkhardt, M. C. McCann and P. Ulvskov. 2002. Direct interference with rhamnogalacturonan I biosynthesis in golgi vesicles. Plant Physiol. 129: 95-102.

Stratilova, E., O. Markovic, M. Dzurova, A. Malovikova, P. Capek and J. Omelkova. 1998. The pectolytic enzymes of carrots. Biolo. Bratisl. 53: 731-738. 
Trillo-Hernández, E. A. 2016. Changes in the Expression of Genes Encoding Isoenzymes of Rhamnogalacturonan Lyase during the Ontogeny of Tomato Fruit (Solanum lycopersicum L.). $13^{\text {th }}$ Annual Solanaceae Conference SolGenomics: From Advances to Applications, Davis, California, USA.

Vicente, A. R., M. Saladié, J. K. C. Rose and J. M. Labavitch. 2007. The linkage between cell wall metabolism and fruit softening: Looking to the future. J. Sci. Food Agric. 87: 1435-1448.

Vincken, J. P., H. A. Schols, R. J. F. Oomen, M. C. McCann, P. Ulvskov,
A. G. J. Voragen and R. G. F. Visser. 2003. If homogalacturonan were a side chain of rhamnogalacturonan I. Implications for cell wall architecture. Plant Physiol. 132: 1781-1789.

Willats, W. G., L. McCartney and J. P. Knox. 2001. In-situ analysis of pectic polysaccharides in seed mucilage and the root surface of Arabidopsis thaliana. Planta. 213: 37-44.

Yapo, B. M. 2011. Rhamnogalacturonan-I: A structurally puzzling and functionally versatile polysaccharide from plant cell walls and mucilages. Polym. Rev. 51: 391-413. 